ARTICLE

https://doi.org/10.1038/s41467-019-12036-5

\title{
A termination-insensitive and robust electron gas at the heterointerface of two complex oxides
}

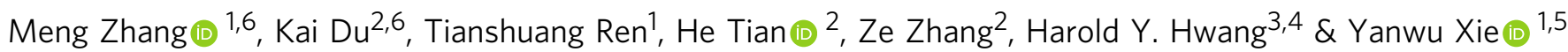

The single-crystal $\mathrm{SrTiO}_{3}$ (001) has two different surface terminations, $\mathrm{TiO}_{2}$ and $\mathrm{SrO}$. One most remarkable observation in previous studies is that only the heterointerfaces with $\mathrm{TiO}_{2}$ terminated $\mathrm{SrTiO}_{3}$, which usually combines with polar oxides such as $\mathrm{LaAlO}_{3}$, host an electron gas. Here we show that a robust electron gas can be generated between a non-polar oxide, $\mathrm{CaHfO}_{3}$, and $\mathrm{SrTiO}_{3}$ (001) with either termination. Unlike the well-known electron gas of $\mathrm{LaAlO}_{3} / \mathrm{SrTiO}_{3}$, the present one of $\mathrm{CaHfO}_{3} / \mathrm{SrTiO}_{3}$ essentially has no critical thickness of $\mathrm{CaHfO}_{3}$, can survive a long-time oxygen annealing at high temperature, and its transport properties are stable under exposure to water and other polar solvents. By electrostatic gating through $\mathrm{CaHfO}_{3}$, field-effect devices are demonstrated using $\mathrm{CaHfO}_{3} / \mathrm{SrTiO}_{3}$ heterointerfaces with both terminations. These results show that the electron gas reported in the present study is unique and promising for applications in oxide electronics.

\footnotetext{
${ }^{1}$ Interdisciplinary Center of Quantum Information, Zhejiang Province Key Laboratory of Quantum Technology and Device, and Department of Physics, Zhejiang University, 310027 Hangzhou, China. ${ }^{2}$ Center of Electron Microscopy, State Key Laboratory of Silicon Materials, and School of Materials Science and Engineering, Zhejiang University, 310027 Hangzhou, China. ${ }^{3}$ Geballe Laboratory for Advanced Materials, Department of Applied Physics, Stanford University, Stanford, CA 94305, USA. ${ }^{4}$ Stanford Institute for Materials and Energy Sciences, SLAC National Accelerator Laboratory, Menlo Park, CA 94025, USA. ${ }^{5}$ Collaborative Innovation Center of Advanced Microstructures, Nanjing University, 210093 Nanjing, China. ${ }^{6}$ These authors contributed equally: Meng Zhang, Kai Du. Correspondence and requests for materials should be addressed to H.T. (email: hetian@zju.edu.cn) or to Y.X. (email: ywxie@zju.edu.cn)
} 
S $\mathrm{rTiO}_{3}$ (STO) is the workhorse oxide semiconductors. Since the discovery of the high-mobility electron gas (EG) at the $\mathrm{LaAlO}_{3} /$ STO heterointerface ${ }^{1}$, much effort has been undertaken to explore and understand the emergent phenomena at this heterointerface ${ }^{2}$. Many intriguing properties that are not present in conventional semiconductor heterostructures, such as interface magnetism ${ }^{3,4}$, interface superconductivity ${ }^{5,6}$, and even the coexistence of magnetism and superconductivity at the interface ${ }^{7,8}$, have been observed. Applications in field-effect transistors (FETs) ${ }^{9-11}$ and integrated circuit devices ${ }^{12}$ have also been demonstrated. One most remarkable result in previous studies is that the EG can only be formed on $\mathrm{TiO}_{2}$-terminated STO (T-STO), and the heterointerface with SrO-terminated STO (S-STO) is highly insulating ${ }^{1,13}$. This extreme asymmetry originates from the polar arrangement of atomic layers in $\mathrm{LaAlO}_{3}$, and can be understood in an elegant polar discontinuity and electronic reconstruction picture ${ }^{13}$. Inspired by this EG, more EGs have been formed between STO and a few different polar oxides $^{14-19}$. Following a similar idea, very recently a hole gas was achieved in a sandwiched STO/LaAlO 3 /STO heterostructure ${ }^{20,21}$. In fact, nowadays interface engineering with polar oxides has become a very popular designing concept in controlling strongly correlated electrons in transition metal oxides.

However, the properties of EGs involving polar oxides can be dramatically altered by polar adsorbates ${ }^{19,22}$, and thus suffer from ambient water ${ }^{19}$. Alternatively, a few studies suggested that the EGs can be generated without polar oxides (still on T-STO, when STO(001) was used $)^{23,24}$. Particularly, they can be generated by simply capping an amorphous oxide layer on $\mathrm{STO}^{24,25}$. Unfortunately, EGs formed by this way are extremely fragile to oxidation environment ${ }^{19}$. In fact, as far as robustness is concerned, it is rare that these heterointerfaces, no matter formed with polar, non-polar, or amorphous oxides, can be comparable with $\mathrm{LaAlO}_{3} / \mathrm{STO}$ because their EGs quickly vanish after annealing in oxygen at moderate temperatures, i.e. $300{ }^{\circ} \mathrm{C}$ or lower ${ }^{19}$. Until now, it is still a challenge to fabricate an EG that can be robust against environments including ambient water and annealing in oxygen.

In this study, we report on an EG formed at the heterointerface between epitaxial $\mathrm{CaHfO}_{3}(\mathrm{CHO})$ and STO. Distinctly different from all the previous ones, we found that this EG can be formed on S-STO, surprisingly, as well as T-STO, and is extremely robust against environments. $\mathrm{CHO}$ is a non-polar wide bandgap $(\sim 6.2 \mathrm{eV})$ insulator $^{26}$. From the ionic point of view, the cation valences of $\mathrm{CHO}$ is the same as that of STO since in the periodic table of the elements, $\mathrm{Ca}$ and $\mathrm{Hf}$, as well as $\mathrm{Sr}$ and $\mathrm{Ti}$, are respectively in the same columns. Early studies had shown that the interface between amorphous $\mathrm{CHO}$ and STO is metallic ${ }^{27}$. Here we show that the interface between epitaxial CHO and STO can be metallic as well.

\section{Results}

Growth and structural characterizations. Single-crystal films of CHO were deposited on STO substrates using pulsed laser deposition. Details are described in Methods. Figure 1 shows the growth and the structural characterizations of CHO/STO of both terminations. The growth was accurately controlled by in situ monitoring reflection high-energy electron diffraction (RHEED) intensity oscillations (Fig. 1a, b). The S-STO was obtained by inserting a single unit cell (uc) of SrO on T-STO (Fig. 1b). The relatively fast decay of the RHEED intensity oscillations for depositing $\mathrm{CHO}$ on S-STO (Fig. 1b) indicates that the growth deviated slightly from a perfect layer-by-layer mode. However, this imperfection does not affect the quality of $\mathrm{CHO}$ significantly. The atomic force microscopy (AFM) images of the surface of $\mathrm{CHO}$ /
STO of both terminations (Fig. 1c, d) show the steps and terraces of the original substrate surface, indicating high-quality growth. The high quality of the $\mathrm{CHO}$ films can also be appreciated from the X-ray diffraction (XRD) finite-thickness oscillations (Fig. 1e, f). The XRD data (Fig. 1e, f, and Supplementary Fig. 1) reveal epitaxial growth and very high crystallinity. The high-angle annular dark-field scanning transmission electron microscopy (HAADFSTEM) images reveal that the CHO films are coherent with the STO substrates for both terminations (Fig. 1g, h), and the two types of heterointerfaces can be distinguished by the atomic-scale energy-dispersive X-ray spectroscopy (EDS) elemental mapping (Fig. 1i, j).

Electrical transport properties. Figure $2 \mathrm{a}, \mathrm{b}$ shows the temperature dependence of the sheet resistance $R_{\text {sheet }}$ for the asgrown $\mathrm{CHO} / \mathrm{STO}$ heterointerfaces with the thickness of $\mathrm{CHO}$ varying from 1 to $30 \mathrm{uc}$. One important observation is that, unlike all the known heterointerfaces such as $\mathrm{LaAlO}_{3} / \mathrm{STO}^{1,13}$, in which only those prepared on the T-STO show interfacial conductivity, the $\mathrm{CHO} / \mathrm{STO}$ heterointerfaces of both terminations show very high conductivity. Our control experiments demonstrated that the heterointerface of $\mathrm{LaAlO}_{3} / \mathrm{S}$-STO prepared under the same growth condition as CHO/S-STO is highly insulating (see Supplementary Fig. 2), and the CHO/STO heterointerface grown on unterminated STO substrate is still conducting (see Supplementary Fig. 3). These observations confirm that the observed conducting interface of $\mathrm{CHO} / \mathrm{S}-\mathrm{STO}$ is not caused by any incompleteness of the $\mathrm{SrO}$ coverage. We also confirm that the STO substrates underwent the same growth condition, but without film growth and post annealing are highly insulating (beyond our measurement limit). Hall effect measurement reveals that for $\mathrm{CHO} / \mathrm{STO}$ of both terminations, the mobile carriers are electrons (see Supplementary Fig. 4). With increasing the thickness of $\mathrm{CHO}, R_{\text {sheet }}$ shows a general, although not monotonic, trend to decrease (see Fig. 2a, b and Supplementary Fig. 5a, b). Similar observation was reported on $\mathrm{CaZrO}_{3} / \mathrm{STO}$ previously ${ }^{16}$. We found that this trend becomes less obvious for the annealed samples (see Supplementary Fig. 5c, d), suggesting that it is related to the $\mathrm{CHO}$-thickness-dependent amount of oxygen vacancies in STO.

Another interesting observation is that, for $\mathrm{CHO} / \mathrm{STO}$ with both terminations, although their conductivities change with the thickness of $\mathrm{CHO}$, there is still measurable conductivity at room temperature even when the thickness of $\mathrm{CHO}$ is as thin as $1 \mathrm{uc}$ (see Fig. 2a, b and Supplementary Figs. 6a, b and 7). We found that the room-temperature conductivity of $1 \mathrm{uc} \mathrm{CHO} / \mathrm{STO}$ is remarkably stable and showed no obvious aging after 1-year storage. By contrast, when the thickness of $\mathrm{LaAlO}_{3}$ is $3 \mathrm{uc}$ or below, the $\mathrm{LaAlO}_{3} / \mathrm{STO}$ heterointerface is highly insulating (see Supplementary Fig. 2) ${ }^{28}$. Therefore, there is no strict critical thickness in CHO/STO. These two observations indicate that the EG observed at the $\mathrm{CHO} / \mathrm{STO}$ heterointerfaces is different from all the previous ones ${ }^{1,15-19,23}$.

The EG of CHO/STO is robust against annealing in oxygen (Fig. 2c-f, Supplementary Figs. 6 and 7). Previous studies ${ }^{19,29}$ had shown that the electrical conductivity and behavior of all the oxide EGs can be strongly suppressed by annealing in oxygen, because annealing will remove oxygen vacancies in both the STO substrates and the films. Most previously reported EGs cannot survive an annealing in oxygen at $300{ }^{\circ} \mathrm{C}$ or even much lower ${ }^{19}$. In contrast, Fig. $2 \mathrm{c}$, d shows that the $\mathrm{CHO} / \mathrm{STO}$ can remain metallic over the whole temperature range (or above $100 \mathrm{~K}$ ) after an in situ annealing at a temperature of $500^{\circ} \mathrm{C}$ (or $\left.600{ }^{\circ} \mathrm{C}\right)$, under partial pressure $P\left(\mathrm{O}_{2}\right)=200 \mathrm{mbar}$. This robustness is close to that of $\mathrm{LaAlO}_{3} / \mathrm{T}$-STO. Furthermore, we found that even after a 24-h 
a

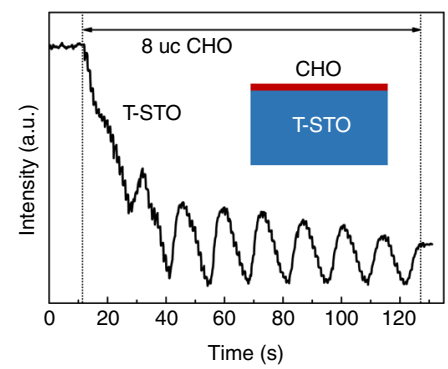

b

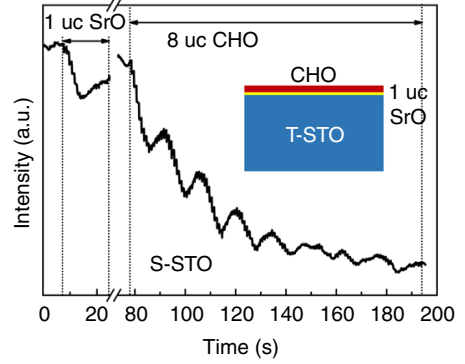

C

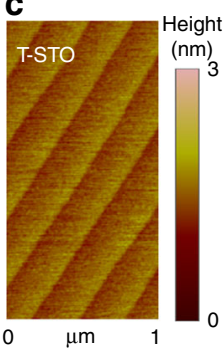

d

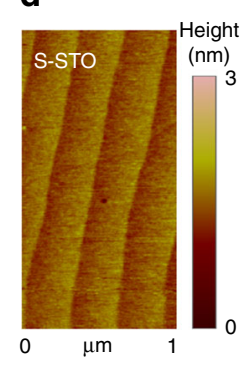

e

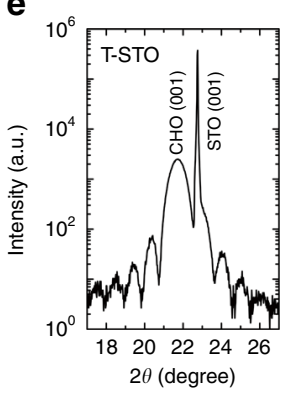

f

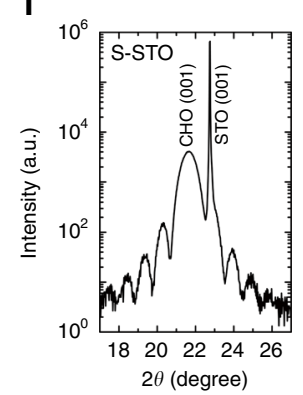

i

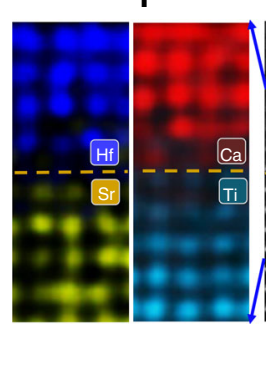

g

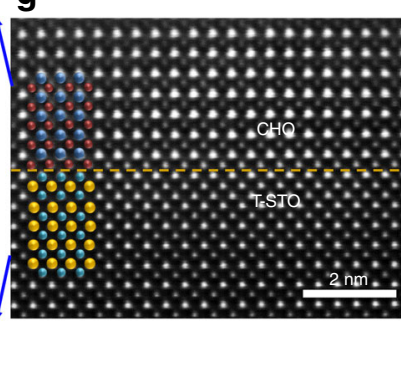

h

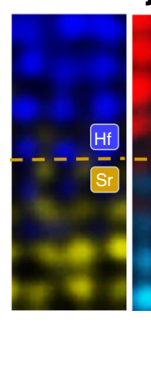

j

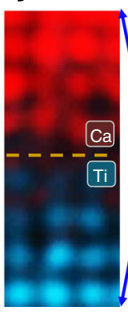

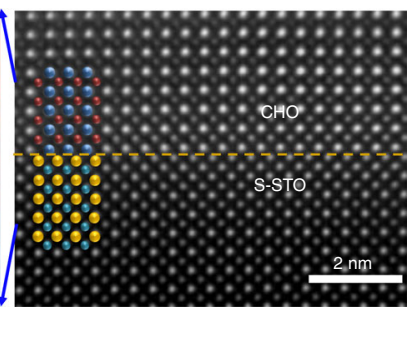

Fig. 1 Growth and structural characterizations of two types of interfaces between $\mathrm{CaHfO}_{3}(\mathrm{CHO})$ and $\mathrm{SrTiO}_{3}(\mathrm{STO})$. a, b Reflection high-energy electron

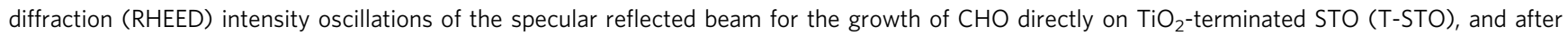
a monolayer of SrO was deposited on the T-STO, namely SrO-terminated STO (S-STO), respectively. Insets show the schematics of the two structures. c, $\mathbf{d}$ Atomic force microscopy (AFM) surface morphologies of 8 uc CHO grown on T-STO and S-STO, respectively. The size of both images is $1 \mu \mathrm{m} \times 2 \mu \mathrm{m}$. e, $\mathbf{f}$ X-ray diffraction (XRD) finite-thickness oscillations in the vicinity of the (001) reflection for 20 uc CHO grown on T-STO and S-STO, respectively. $\mathbf{g}$, $\mathbf{h}$ Cross-sectional scanning transmission electron microscopy (STEM) images of 8 uc CHO grown on T-STO and S-STO, respectively. $\mathbf{i}, \mathbf{j}$ The energydispersive $\mathrm{X}$-ray spectroscopy (EDS) elemental mapping of the same samples from $\mathbf{g}$, $\mathbf{h}$, respectively

annealing at $1000^{\circ} \mathrm{C}$, under $P\left(\mathrm{O}_{2}\right)=1 \mathrm{bar}$, the $\mathrm{CHO} / \mathrm{STO}$ can still remain metallic down to $150 \mathrm{~K}$ (Fig. 2e, f). Note that our control experiments showed that even $\mathrm{LaAlO}_{3} / \mathrm{T}$-STO became highly insulating after the same extreme annealing (see Supplementary Fig. 9).

Besides its robustness to the annealing in oxygen, the presently observed EG of CHO/STO shows a few other interesting features. First, the CHO/S-STO generally has even better electrical transport properties than the CHO/T-STO (Fig. 2). Second, the electron mobility of $\mathrm{CHO} / \mathrm{STO}$ is high. As shown in Fig. 2 and Supplementary Figs. 4, 6, and 7, for the metallic CHO/STO, the residual resistivity ratio $\left(R_{\text {sheet }}(300 \mathrm{~K}) / R_{\text {sheet }}(2 \mathrm{~K})\right)$ can be more than three orders of magnitude, indicating a very high mobility. Hall effect measurement reveals that the low-temperature mobility can reach $10,000 \mathrm{~cm}^{2} / \mathrm{V} / \mathrm{s}$ (Supplementary Fig. 4c), comparable with that of the best prepared $\mathrm{LaAlO}_{3} / \mathrm{T}-\mathrm{STO}$ samples ${ }^{30}$. Third, unlike $\mathrm{LaAlO}_{3} / \mathrm{T}-\mathrm{STO}{ }^{19,22}$, the EG of CHO/ STO is extremely stable against surface exposure of polar solvents. We treated the $\mathrm{CHO} / \mathrm{STO}$ samples, prepared under various conditions, with water and other polar solvents as we did previously for EGs at other heterointerfaces ${ }^{19,22}$, and found negligible influence on their conductivity (see Supplementary Fig. 10 and Supplementary Table 1). This point is important for device applications because it assures that the performance of devices can be stable under different environments.

Possible origin of the EG of CHO/STO. We now consider the possible origin of the $\mathrm{EG}$ of $\mathrm{CHO} / \mathrm{STO}$. Since $\mathrm{CHO}$, unlike $\mathrm{LaAlO}_{3}$, is non-polar, one can exclude the classic polar discontinuity and electronic reconstruction scenario ${ }^{13}$. An alternative plausible scenario is the strain-induced polarization and electronic reconstruction, as proposed by a theoretical study ${ }^{31}$ and experimentally observed at the heterointerface of $\mathrm{CaZrO}_{3} /$ $\mathrm{STO}^{16}$. However, the B-site atom displacement vector maps of
CHO/STO (see Supplementary Fig. 11) show that the atom displacements are tiny and random, and the resulting net out-ofplane polarization is within the detection error of our STEM measurement. Furthermore, the absence of a critical thickness and the inertness to polar solvents are unexpected from a straininduced polarization mechanism. Therefore, we conclude that the EG of CHO/STO is unlikely to originate from the strain-induced polarization in $\mathrm{CHO}$ films.

One other possible cause is the oxygen non-stoichiometry. Experimental data show that the overall conductivity decreases with increasing partial oxygen pressure during growth (see Supplementary Fig. 8), and the overall conductivity increases with increasing thickness of CHO (Fig. 2a, b and Supplementary Fig. 5). These findings suggest that oxygen non-stoichiometry in the $\mathrm{CHO}$ films (rather than the STO substrate) plays an important role in the formation of EG. The EG may be formed due to the oxygen non-stoichiometry in the $\mathrm{CHO}$ films, by transferring electrons to the interfacial STO ${ }^{19,32,33}$ or by generating oxygen vacancies in the interfacial STO via oxygen migration ${ }^{34,35}$. A remaining puzzle is why the EG of CHO/STO can survive such a severe annealing in oxygen. One interesting possibility is that the existence of some oxygen vacancies in $\mathrm{CHO}$ or the interface of $\mathrm{CHO} / \mathrm{STO}$ is thermodynamically stable, similar to the cases of the surface of $\mathrm{LaAlO}_{3} / \mathrm{T}-\mathrm{STO}^{32}$, the interface of $\mathrm{LaAlO}_{3} / \mathrm{S}_{-} \mathrm{STO}^{13}$, or the interface of $\gamma-\mathrm{Al}_{2} \mathrm{O}_{3} / \mathrm{T}-\mathrm{STO}^{23}$.

Device application. $\mathrm{CHO}$ has an orthorhombic structure with a pseudocubic lattice constant of $3.990 \AA$ A; STO has a cubic lattice constant of $3.905 \AA$. The good matching of their lattices makes $\mathrm{CHO}$ a natural gating and insulating material for STO-based oxide devices ${ }^{36}$. Early studies from Lippmaa group ${ }^{37,38}$ had shown that both the crystalline and the amorphous $\mathrm{CHO}$ films grown on STO exhibit excellent dielectric properties with a relative dielectric constant around 17, comparable with $\mathrm{LaAlO}_{3}$ or 
a

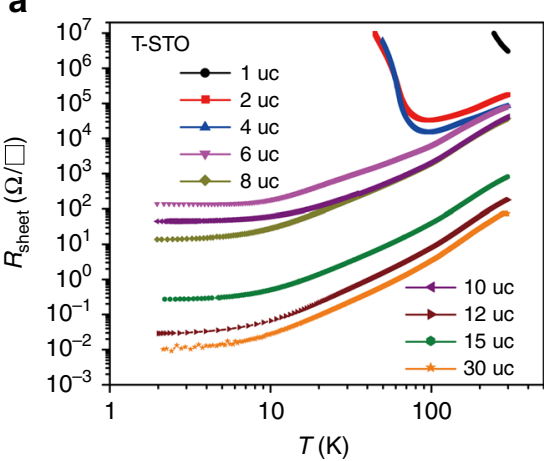

b

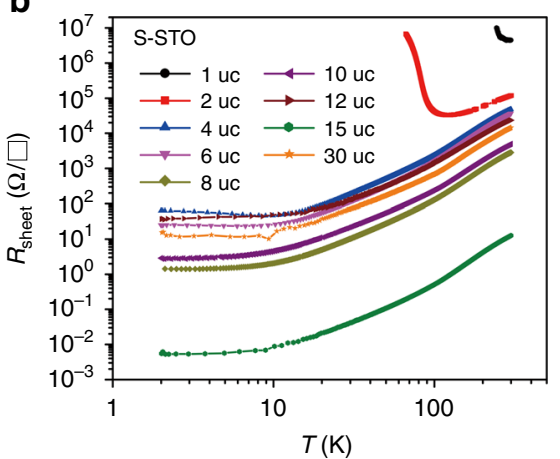

C

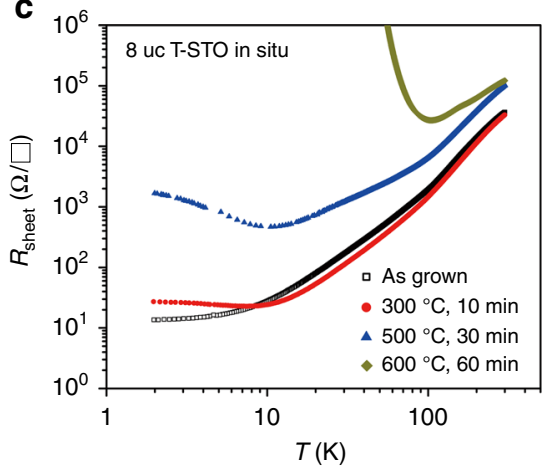

d

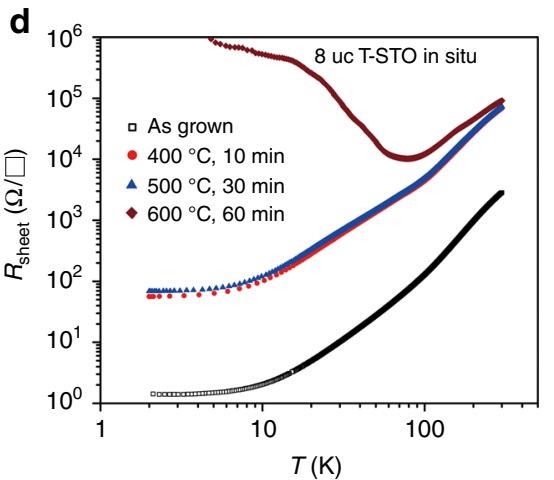

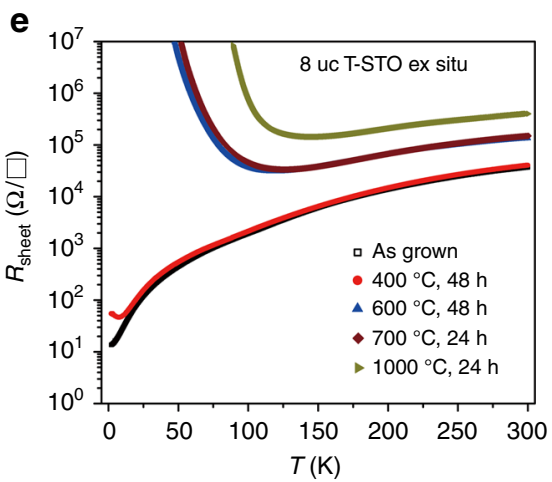

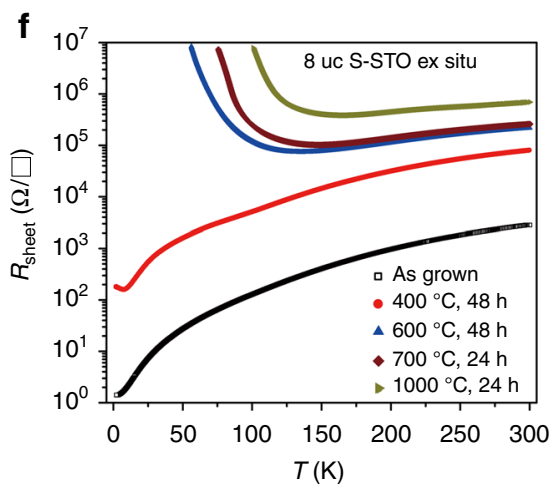

Fig. 2 Electrical transport properties of $\mathrm{CaHfO}_{3} / \mathrm{SrTiO}_{3}(\mathrm{CHO} / \mathrm{STO})$ heterointerfaces. $\mathbf{a}$, $\mathbf{b}$ Temperature dependence of $R_{\text {sheet }}$ for the as-grown $\mathrm{CHO}$ of various thicknesses grown on $\mathrm{TiO}_{2}$-terminated STO (T-STO) and SrO-terminated STO (S-STO), respectively. The same samples were post-annealed ex situ in $\mathrm{O}_{2}$, and the corresponding results are shown in Supplementary Fig. 6. c, d Temperature dependence of $R_{\text {sheet }}$ for the in situ annealed CHO (8 uc) grown on T-STO and S-STO, respectively. Each curve represents a sample that has been annealed in situ under $P\left(\mathrm{O}_{2}\right)=200 \mathrm{mbar}$, at the conditions as labeled. More in situ annealing results on samples of different thicknesses are presented in Supplementary Fig. 7. e, $\mathbf{f}$ Temperature dependence of $R_{\text {sheet }}$ for the ex situ annealed CHO (8 uc) grown on T-STO and S-STO, respectively. In each case, one as-grown sample was annealed ex situ subsequently from low to high annealing temperature, under $P\left(\mathrm{O}_{2}\right)=1 \mathrm{bar}$, in a tube furnace. For clarity, double-logarithmic coordinates were used in a-d, and semi-logarithmic coordinates were used in $\mathbf{e}, \mathbf{f}$

a

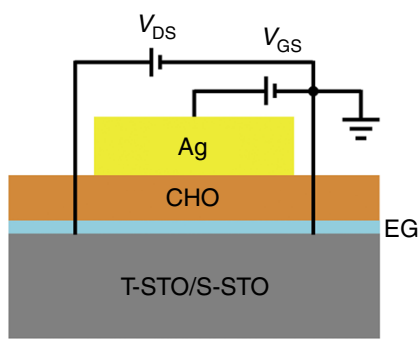

b

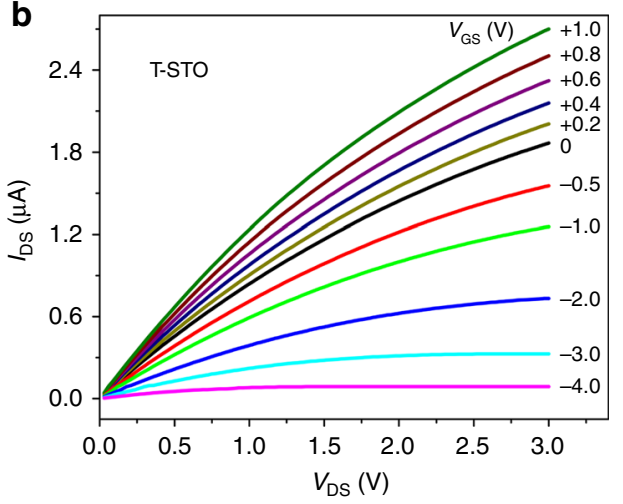

C

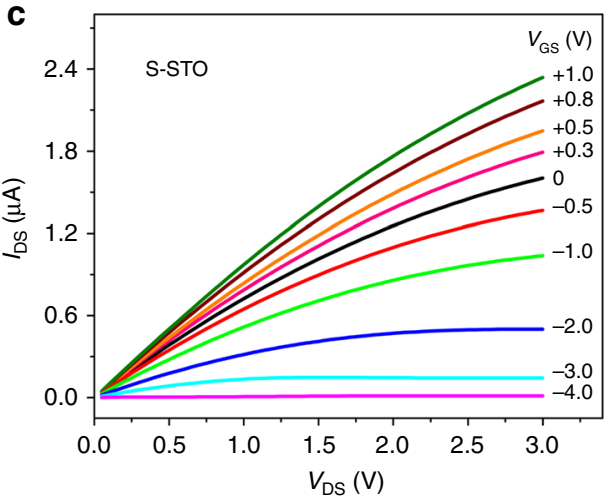

Fig. 3 Field-effect transistors with in situ annealed 8-uc $\mathrm{CaHfO}_{3} / \mathrm{SrTiO}_{3}(\mathrm{CHO} / \mathrm{STO})$ heterointerfaces. a Sketch of a device. The channel is $10 \mu \mathrm{m}$ in width and $100 \mu \mathrm{m}$ in length. The top gate electrode is a 100-nm-thick silver film. Drain-source current $\left(I_{D S}\right)$ versus drain-source voltage $\left(V_{D S}\right)$ at different gatesource voltages $\left(V_{G S}\right)$ for $\mathbf{b}$ CHO grown on $\mathrm{TiO}_{2}$-terminated STO (T-STO) and $\mathbf{c}$ SrO-terminated STO (S-STO). All data were measured at room temperature

$\mathrm{HfO}_{2}$ (note that $\mathrm{HfO}_{2}$ is replacing $\mathrm{SiO}_{2}$ as the gating and insulating material in commercial integrated circuits). Here, we demonstrate that the $\mathrm{CHO} / \mathrm{STO}$ of both terminations can be used to fabricate field-effect devices.

As schematically shown in Fig. 3a, the EG at the CHO/STO heterointerface is used as the drain (D)-source (S) channel, and the epitaxial $\mathrm{CHO}$ film as gate dielectrics, for FET operations. The output characteristics, drain-source current $\left(I_{\mathrm{DS}}\right)$ versus drainsource voltage $\left(V_{\mathrm{DS}}\right)$ at different gate voltages $\left(V_{\mathrm{GS}}\right)$, are shown in
Fig. 3b, c. Note that although the thickness of CHO is only $8 \mathrm{uc}$ $(\sim 3.2 \mathrm{~nm})$ (similar FETs with $15 \mathrm{uc} \mathrm{CHO}$ are shown in Supplementary Fig. 13), in the $-4 \mathrm{~V}<V_{\mathrm{GS}}<1 \mathrm{~V}$ range, the leakage current of the devices is very small (see Supplementary Fig. 12). In addition, we found that even increasing the size of the contact between the gate electrode and the $\mathrm{CHO}$ film up to several millimeter square, in the same $V_{\mathrm{GS}}$ range the leakage current remains small (not shown), confirming that the $\mathrm{CHO}$ film of $\mathrm{CHO} / \mathrm{STO}$ is very homogeneous. 


\section{Discussion}

The present study demonstrates that a robust EG can be generated at the heterointerface of an epitaxially grown $\mathrm{CHO}$ film and a STO substrate with either termination. This EG is distinctly different from that of $\mathrm{LaAlO}_{3} / \mathrm{T}$-STO because it is insensitive to the termination of STO, has no strict critical thickness of $\mathrm{CHO}$, and is stable against polar adsorbates. This EG is also remarkably stable against annealing in oxygen, comparable with that of $\mathrm{LaAlO}_{3} / \mathrm{T}-\mathrm{STO}$. Using this EG as the conducting channel, and $\mathrm{CHO}$ as gating dielectrics, FETs are constructed. The highperformance gating ability, integrated with the robustness to environments (temperature, oxygen, humidity, and solvents), makes the EG at the interfaces of $\mathrm{CHO} / \mathrm{STO}$ very attractive for applications in oxide electronics.

\section{Methods}

Material. CHO has an orthorhombic structure with $a=5.719, b=7.982$, and $c=$ $5.5578 \AA^{39}$. The pseudocubic lattice constant is $3.990 \AA$. STO has a cubic lattice constant of $3.905 \AA$. The average lattice mismatch between them is $+2.3 \%{ }^{39}$. The XRD reciprocal space maps (Supplementary Fig. 1c, d) indicate that for both terminations of STO, the CHO films are constrained on the STO substrates, with an in-plane lattice constant of $3.905 \AA$ and out-of-plane lattice constant of $4.075 \AA$ for $\mathrm{CHO} / \mathrm{T}$-STO, and $4.102 \AA$ for $\mathrm{CHO} / \mathrm{S}$-STO.

Pulsed laser deposition synthesis. T-STO substrates were pre-annealed in situ at an oxygen partial pressure $P\left(\mathrm{O}_{2}\right)$ of $1 \times 10^{-4}$ mbar for $20 \mathrm{~min}$ at a temperature of $975^{\circ} \mathrm{C}$ to achieve sharp step-and-terrace surfaces. S-STO substrates were prepared by depositing 1 uc SrO layer on the pre-annealed T-STO substrates in situ at a temperature of $800^{\circ} \mathrm{C}$, and $P\left(\mathrm{O}_{2}\right)=1 \times 10^{-4} \mathrm{mbar}$, using $1.5 \mathrm{~J} / \mathrm{cm}^{2}$ laser fluence. A 248-nm $\mathrm{KrF}$ excimer laser was used for the deposition. All the $\mathrm{CHO}$ films presented in this work were grown at a substrate temperature $T_{\mathrm{g}}$ of $800^{\circ} \mathrm{C}$, and $P\left(\mathrm{O}_{2}\right)=1 \times 10^{-4} \mathrm{mbar}$, using $3 \mathrm{~J} / \mathrm{cm}^{2}$ laser fluence. After growth, the samples without in situ post-annealing were cooled down at a rate of $100^{\circ} \mathrm{C} / \mathrm{min}$ under the growth oxygen pressure; the samples with in situ post-annealing were cooled down at a rate of $100^{\circ} \mathrm{C} / \mathrm{min}$ to $500^{\circ} \mathrm{C}$, stayed at $500^{\circ} \mathrm{C}$ for 30 min under $P\left(\mathrm{O}_{2}\right)=200$ mbar, and then cooled down to room temperature under the same oxygen pressure. The CHO polycrystalline target was prepared by sintering a mixture of stoichiometric amounts of $\mathrm{CaCO}_{3}$ and $\mathrm{HfO}_{2}$ at $1300^{\circ} \mathrm{C}$ for $12 \mathrm{~h}$.

Structural characterizations. The AFM images were acquired in tapping mode with Veeco DI3100. The XRD data were taken using a monochromated $\mathrm{Cu}-\mathrm{K}_{\alpha}$ source on a Bruker AXS D8-Discover. Cross-sectional specimens for STEM investigations were prepared by a FEI Quanta 3D FEG Focused Ion Beam. STEM images were acquired using a spherical aberration-corrected microscope equipped with four Super-X EDS detectors (FEI Titan G2 80-200 Chemi STEM, 30 mrad convergence angle).

Electrical transport measurement. The conducting $\mathrm{CHO} / \mathrm{STO}$ interfaces were contacted by ultrasonic bonding with $\mathrm{Al}$ wires. The conductivity measurements were performed using a standard four-probe method. The Hall effect measurements were performed using a Hall-bar sample made with $\mathrm{Al}_{2} \mathrm{O}_{x}$ hard mask ${ }^{30}$.

\section{Data availability}

The data that support the findings of this study are available from the corresponding authors upon reasonable request.

Received: 12 March 2019 Accepted: 13 August 2019

Published online: 06 September 2019

\section{References}

1. Ohtomo, A. \& Hwang, H. Y. A high-mobility electron gas at the $\mathrm{LaAlO}_{3} /$ $\mathrm{SrTiO}_{3}$ heterointerface. Nature 427, 423-427 (2004).

2. Hwang, H. Y. et al. Emergent phenomena at oxide interfaces. Nat. Mater. 11, 103-113 (2012).

3. Brinkman, A. et al. Magnetic effects at the interface between non-magnetic oxides. Nat. Mater. 6, 493-496 (2007).

4. Ariando et al. Electronic phase separation at the $\mathrm{LaAlO}_{3} / \mathrm{SrTiO}_{3}$ interface. Nat. Commun. 2, 188 (2011).

5. Reyren, N. et al. Superconducting interfaces between insulating oxides. Science 317, 1196-1199 (2007).
6. Caviglia, A. D. et al. Electric field control of the $\mathrm{LaAlO}_{3} / \mathrm{SrTiO}_{3}$ interface ground state. Nature 456, 624-627 (2008).

7. Li, L., Richter, C., Mannhart, J. \& Ashoori, R. C. Coexistence of magnetic order and two-dimensional superconductivity at $\mathrm{LaAlO}_{3} / \mathrm{SrTiO}_{3}$ interfaces. Nat. Phys. 7, 762-766 (2011).

8. Bert, J. A. et al. Direct imaging of the coexistence of ferromagnetism and superconductivity at the $\mathrm{LaAlO}_{3} / \mathrm{SrTiO}_{3}$ interface. Nat. Phys. 7, 767-771 (2011).

9. Förg, B., Richter, C. \& Mannhart, J. Field-effect devices utilizing $\mathrm{LaAlO}_{3}$ $\mathrm{SrTiO}_{3}$ interfaces. Appl. Phys. Lett. 100, 053506 (2012).

10. Cen, C., Thiel, S., Mannhart, J. \& Levy, J. Oxide nanoelectronics on demand Science 323, 1026-1030 (2009).

11. Woltmann, C. et al. Field-effect transistors with submicrometer gate lengths fabricated from $\mathrm{LaAlO}_{3}-\mathrm{SrTiO}_{3}$-based heterostructures. Phys. Rev. Appl. 4, 064003 (2015).

12. Jany, R. et al. Monolithically integrated circuits from functional oxides. $A d v$. Mater. Interfaces 1, 1300031 (2014).

13. Nakagawa, N., Hwang, H. Y. \& Muller, D. A. Why some interfaces cannot be sharp. Nat. Mater. 5, 204-209 (2006)

14. Huang, Z. et al. The effect of polar fluctuation and lattice mismatch on carrier mobility at oxide interfaces. Nano Lett. 16, 2307-2313 (2016).

15. Hotta, Y., Susaki, T. \& Hwang, H. Y. Polar discontinuity doping of the $\mathrm{LaVO}_{3} /$ $\mathrm{SrTiO}_{3}$ interface. Phys. Rev. Lett. 99, 236805 (2007).

16. Chen, Y. et al. Creation of high mobility two-dimensional electron gases via strain induced polarization at an otherwise nonpolar complex oxide interface. Nano Lett. 15, 1849-1854 (2015).

17. Reinle-Schmitt, M. L. et al. Tunable conductivity threshold at polar oxide interfaces. Nat. Commun. 3, 932 (2012).

18. $\mathrm{Li}, \mathrm{C}$. et al. The metallic interface between insulating $\mathrm{NdGaO}_{3}$ and $\mathrm{SrTiO}_{3}$ perovskites. Appl. Phys. Lett. 103, 201602 (2013).

19. Zhang, M. et al. Origin of interfacial conductivity at complex oxide heterointerfaces: Possibility of electron transfer from water chemistry at surface oxygen vacancies. Phys. Rev. Mater. 2, 065002 (2018).

20. Chen, Y. \& Pryds, N. 2D hole gas seen. Nat. Mater. 17, 215-216 (2018)

21. Lee, H. et al. Direct observation of a two-dimensional hole gas at oxide interfaces. Nat. Mater. 17, 231-236 (2018).

22. Xie, Y., Hikita, Y., Bell, C. \& Hwang, H. Y. Control of electronic conduction at an oxide heterointerface using surface polar adsorbates. Nat. Commun. 2, 494 (2011).

23. Chen, Y. Z. et al. A high-mobility two-dimensional electron gas at the spinel/ perovskite interface of $\gamma-\mathrm{Al}_{2} \mathrm{O}_{3} / \mathrm{SrTiO}_{3}$. Nat. Commun. 4, 1371 (2013).

24. Chen, Y. et al. Metallic and insulating interfaces of amorphous $\mathrm{SrTiO}_{3}$-based oxide heterostructures. Nano Lett. 11, 3774-3778 (2011).

25. Chen, Z. et al. Surface amorphous oxides induced electron transfer into complex oxide heterointerfaces. Adv. Mater. Interfaces 5, 1801216 (2018).

26. Rauwel, E., Galeckas, A., Rauwel, P. \& Fjellvåg, H. Unusual photoluminescence of $\mathrm{CaHfO}_{3}$ and $\mathrm{SrHfO}_{3}$ nanoparticles. Adv. Funct. Mater. 22, 1174-1179 (2012).

27. Shibuya, K., Ohnishi, T., Lippmaa, M. \& Oshima, M. Metallic conductivity at the $\mathrm{CaHfO}_{3} / \mathrm{SrTiO}_{3}$ interface. Appl. Phys. Lett. 91, 232106 (2007).

28. Thiel, S., Hammerl, G., Schmehl, A., Schneider, C. M. \& Mannhart, J. Tunable quasi-two-dimensional electron gases in oxide heterostructures. Science 313, 1942-1945 (2006)

29. Kalabukhov, A., Gunnarsson, R., Olsson, E., Claeson, T. \& Winkler, D. Effect of oxygen vacancies in the $\mathrm{SrTiO}_{3}$ substrate on the electrical properties of the $\mathrm{LaAlO}_{3} / \mathrm{SrTiO}_{3}$ interface. Phys. Rev. B 75, 121404 (2007).

30. Xie, Y., Bell, C., Hikita, Y., Harashima, S. \& Hwang, H. Y. Enhancing electron mobility at the $\mathrm{LaAlO}_{3} / \mathrm{SrTiO}_{3}$ interface by surface control. Adv. Mater. 25, 4735-4738 (2013)

31. Cheng, J., Nazir, S. \& Yang, K. First-principles prediction of two-dimensional electron gas driven by polarization discontinuity in nonpolar/nonpolar $\mathrm{AHfO}_{3} / \mathrm{SrTiO}_{3}(\mathrm{~A}=\mathrm{Ca}, \mathrm{Sr}$, and $\mathrm{Ba})$ heterostructures. ACS Appl. Mater. Interfaces 8, 31959-31967 (2016).

32. Yu, L. \& Zunger, A. A polarity-induced defect mechanism for conductivity and magnetism at polar-nonpolar oxide interfaces. Nat. Commun. 5, 5118 (2014).

33. Scheiderer, P. et al. Surface-interface coupling in an oxide heterostructure impact of adsorbates on $\mathrm{LaAlO}_{3} / \mathrm{SrTiO}_{3}$. Phys. Rev. B 92, 195422 (2015).

34. Chen, Y. et al. Tuning the two-dimensional electron liquid at oxide interfaces by buffer-layer-engineered redox reactions. Nano Lett. 17, 7062-7066 (2017).

35. Nazir, S., Pulikkotil, J. J., Singh, N. \& Schwingenschlögl, U. Vacancy induced metallicity at the $\mathrm{CaHfO}_{3} / \mathrm{SrTiO}_{3}$ interface. Appl. Phys. Lett. 98, 133114 (2011).

36. Feteira, A., Sinclair, D. C., Rajab, K. Z. \& Lanagan, M. T. Crystal structure and microwave dielectric properties of alkaline- earth hafnates, $\mathrm{AHfO}_{3}(\mathrm{~A}=\mathrm{Ba}$, Sr, Ca). J. Am. Ceram. Soc. 91, 893-901 (2008). 
37. Shibuya, K., Ohnishi, T., Lippmaa, M., Kawasaki, M. \& Koinuma, H. Single crystal $\mathrm{SrTiO}_{3}$ field-effect transistor with an atomically flat amorphous $\mathrm{CaHfO}_{3}$ gate insulator. Appl. Phys. Lett. 85, 425-427 (2004).

38. Shibuya, K. et al. Field-effect modulation of the transport properties of nondoped $\mathrm{SrTiO}_{3}$. Appl. Phys. Lett. 88, 212116 (2006).

39. Shibuya, K., Ohnishi, T., Lippmaa, M., Kawasaki, M. \& Koinuma, H. Domain structure of epitaxial $\mathrm{CaHfO}_{3}$ gate insulator films on $\mathrm{SrTiO}_{3}$. Appl. Phys. Lett. 84, 2142-2144 (2004).

\section{Acknowledgements}

We greatly thank Dr. Hongrui Zhang, Prof. Jirong Sun, and Prof. Baogen Shen for the help in XRD measurements. This work was supported by the National Key R\&D Program of the MOST of China (Grants No. 2016YFA0300204, No. 2017YFA0303002), and the Fundamental Research Funds for the Central Universities.

\section{Author contributions}

M.Z. and T.R. fabricated and characterized the heterointerfaces. K.D., H.T. and Z.Z. measured and analyzed the STEM data. H.Y.H. guided the work. Y.X. designed the experiment and wrote the manuscript, with input from all authors.

\section{Additional information}

Supplementary Information accompanies this paper at https://doi.org/10.1038/s41467019-12036-5.

Competing interests: The authors declare no competing interests.
Reprints and permission information is available online at http://npg.nature.com/ reprintsandpermissions/

Peer review information: Nature Communications thanks Yunzhong Chen and other anonymous reviewers for their contribution to the peer review of this work. Peer reviewer reports are available.

Publisher's note: Springer Nature remains neutral with regard to jurisdictional claims in published maps and institutional affiliations.

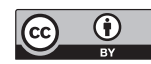

Open Access This article is licensed under a Creative Commons Attribution 4.0 International License, which permits use, sharing, adaptation, distribution and reproduction in any medium or format, as long as you give appropriate credit to the original author(s) and the source, provide a link to the Creative Commons license, and indicate if changes were made. The images or other third party material in this article are included in the article's Creative Commons license, unless indicated otherwise in a credit line to the material. If material is not included in the article's Creative Commons license and your intended use is not permitted by statutory regulation or exceeds the permitted use, you will need to obtain permission directly from the copyright holder. To view a copy of this license, visit http://creativecommons.org/ licenses/by/4.0/.

(C) The Author(s) 2019 